\title{
Quantitative Analysis of a Directed Behavior in the Medicinal Leech: Implications for Organizing Motor Output
}

\author{
John E. Lewis and William B. Kristan Jr \\ Department of Biology, University of California, San Diego, La Jolla, California 92093-0357
}

The local bend is a directed behavior produced by the leech, Hirudo medicinalis, in response to a light touch. Contraction of longitudinal muscles near the touched location results in a bend directed away from the stimulus. We quantify the relationship between the location of touch around the body perimeter and the behavioral output by using video analysis, muscle tension measurements, and electromyography. On average, the direction of the behavioral output differed from the touch location by $<8 \%$ of the total body perimeter. We discuss our results in the context of two contrasting behavioral strategies: a Continuous strategy, in which the local bend is directed exactly opposite to stimulus location, and a Categorical strategy, in which there are four distinct bend directions, each elicited by stimuli given in a single quadrant of the body perimeter. To distinguish between these strategies, we delivered two competing stimuli simulta- neously. The resulting behavioral output is best described by an average of the effects of each stimulus given alone and thus provides support for the Continuous strategy. We also use a simple model, based on anatomical and physiological data, to predict the responses of the known motor neurons to different stimulus locations. The model shows that the activation of two of the motor neurons ( $D$ and $V$ ) is inconsistent with a Categorical strategy. However, these neurons are known to be active during the local bend behavior. This result, along with our experimental observations, suggests that the local bend network uses a Continuous strategy to encode stimulus location and produce directed behavioral output.

Key words: behavioral accuracy; Hirudo medicinalis; local bend; population coding; sensorimotor transformation; touch
Information processing in sensorimotor networks often involves population codes in which the distributed activity of broadly tuned neurons represents a specific sensory or motor parameter. Current understanding of population coding has resulted mainly from studies of directed behaviors (Churchland and Sejnowski, 1992; Katz, 1996). Directed behaviors transform a stimulus location in space to a directed movement in space, with the underlying neuronal networks mapping a population-coded stimulus representation to a population-coded motor command.

Some directed behaviors, such as orienting toward auditory cues in the barn owl (Knudsen et al., 1979) and saccadic eye movements in primates (Lee et al., 1988), involve a continuous mapping of a sensory stimulus to behavioral output. Other behaviors, such as the scratch reflex in turtles (Stein, 1989) and the tailflip escape response in crayfish (Krasne and Wine, 1984), involve a categorical mapping, such that a range of stimuli produces an identical behavioral response, with sharp borders between ranges of stimuli that produce different responses. Continuous and Categorical strategies predict differences in the organization of motor output. A Continuous strategy could involve population coding at all processing stages, from encoding the sensory stimulus to encoding movement direction. Accurate transfer of population-coded information between processing

Received July 29, 1997; revised Nov. 19, 1997; accepted Nov. 25, 1997.

This work was supported by National Research Service Award Predoctoral Fellowship MH10677 (J.E.L.), National Institutes of Health Training Grant GM08107 (J.E.L.), and National Institutes of Health Research Grant MH43396 (W.B.K.). We thank R. J. A. Wilson for many helpful discussions.

Correspondence should be addressed to Dr. William B. Kristan Jr, Department of Biology 0357, University of California, San Diego, 9500 Gilman Drive, La Jolla, CA, 92093-0357.

Dr. Lewis's present address: Cellular and Molecular Medicine, University of Ottawa, Ottawa, Ontario, Canada K1H-8M5.

Copyright (C) 1998 Society for Neuroscience $\quad 0270-6474 / 98 / 181571-12 \$ 05.00 / 0$ stages could result from synaptic interactions that are governed by relatively simple rules (Salinas and Abbott, 1995). In contrast, a Categorical strategy requires a choice between distinct responses, so at some processing stage, dedication to a single type of behavior must be accomplished, perhaps by using a competitive winnertake-all mechanism (Yuille and Grzywacz, 1989). Directly testing the predictions made by different behavioral strategies has been difficult because of the size and complex organization of the networks involved. In general, it is not possible to study a single system at multiple stages of a sensorimotor transformation. It is useful then to investigate smaller systems and simple behaviors in which it is feasible to study the entire transformation with singleneuron resolution.

One such behavior is the local bend behavior in the medicinal leech (Kristan et al., 1995), which is elicited by a touch to the body wall and results in a bend of the body directed away from the touched site (Fig. 1A). The local bend network resides in a single segmental ganglion and consists of three neuronal levels (Fig. $1 C)$. Two types of mechanosensory neurons, T-cells and P-cells, for touch and pressure, have overlapping receptive fields, such that a given stimulus activates one or two P-cells and up to three T-cells (Nicholls and Baylor, 1968; Lewis, 1997). The longitudinal motor neurons comprise seven distinct classes, five excitatory (Fig. 1C) and two inhibitory (Stuart, 1970; Mason and Kristan, 1982), which innervate longitudinal muscle with overlapping fields.

We characterize the local bend response as a function of touch location to determine whether this behavior uses a Continuous or Categorical strategy. The Continuous strategy involves a different bend direction for every stimulus location. The broadly tuned neurons in the local bend network are well suited for such a strategy. In the Categorical strategy one of four possible bend 


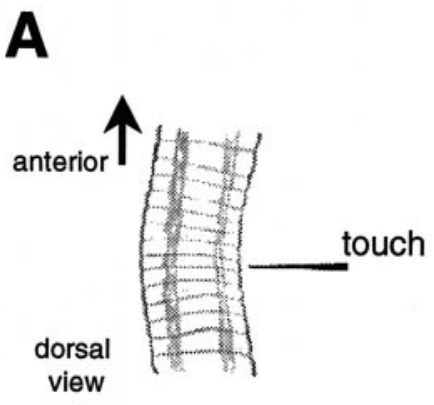

B

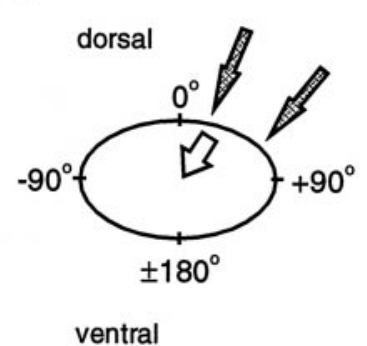

C

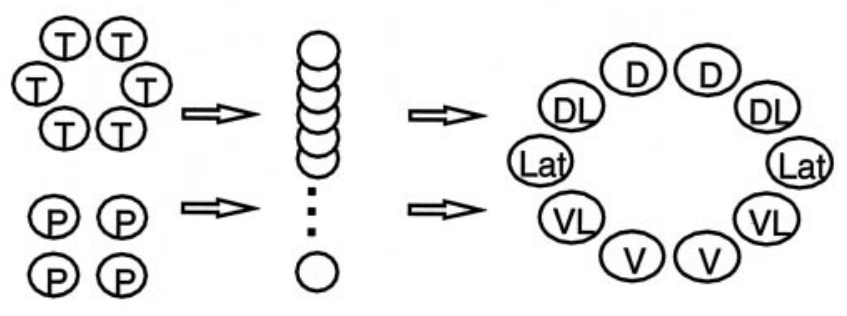

sensory neurons

interneurons

motor neurons

Figure 1. The local bend behavior and network. A, Illustration of three segments of the leech midbody, producing a local bend (dorsal view, anterior is $u p$ ). Each segment consists of five annuli, and each annulus forms a ring around the body perimeter. A touch to the lateral body wall elicits a bend directed away from the touched site. The bend results from shortening of longitudinal muscles near the touched site. $B$, Schematic of the body perimeter in cross section showing the convention for defining body wall location: dorsal midline $\left(\theta=0^{\circ}\right)$, ventral midline $\left(\theta= \pm 180^{\circ}\right)$, right lateral $\left(\theta=+90^{\circ}\right)$, and left lateral $\left(\theta=-90^{\circ}\right)$. Shaded arrows give two examples of different stimulus locations; the unfilled arrow gives an example of a bend direction. $C$, Schematic outline of the local bend neuronal network. Two classes of mechanosensory neurons ( $T$ and $P$ ) connect to a layer of interneurons $(\sim 30$, with 17 identified so far; Lockery and Kristan, 1990b), which in turn connect to five classes of motor neurons that innervate longitudinal muscles in different regions of the body wall: dorsal $(D)$, dorsolateral $(D L)$, lateral (Lat), ventrolateral $(V L)$, and ventral $(V)$. There are also two classes of inhibitory motor neurons (not shown).

directions is produced, depending on stimulus location. Each of the four responses is associated with one of the four P-cells. The $\mathrm{P}$-cell that is activated to the greatest extent determines which response, of the four, is expressed (winner-take-all). We provide evidence, based on experimental and theoretical approaches, that the local bend network uses a Continuous strategy in the sensorimotor transformation of stimulus location to directed behavioral output.

Portions of this work have appeared previously in preliminary form (Lewis and Kristan, 1996).

\section{MATERIALS AND METHODS}

The leech has a body plan consisting of 21 midbody segments (denoted MS1-MS21) with a corresponding segmental nerve cord (one ganglion per segment). This organization is amenable to semi-intact preparations in which parts of the nervous system are exposed for electrophysiology while most of the animal is left intact (Kristan et al., 1974; Muller et al., 1981). Experiments were performed on 1.5-2.5 gm leeches, Hirudo medicinalis, obtained from Leeches USA (Westbury, NY). Animals were anesthetized in ice-cold leech saline, and surgical methods were similar to those described previously (Muller et al., 1981). We used standard intracellular recording techniques with sharp electrodes $(20-30 \mathrm{M} \Omega$ ) and the Axoclamp-2B amplifier (Axon Instruments, Foster City, CA). Neurons were identified on the basis of their physiology and location within the ganglion (Muller et al., 1981). The preparations and behavioral measurement techniques used are described in following sections. Data were collected by the Axotape 2.0 and Digidata 1200 PC-based data acquisition system (Axon Instruments). Off-line data analyses were performed with Axotape 2.0 and Axograph 2.0 (Axon Instruments), Systat 5.2 (Systat, Evanston, IL), and Microsoft Excel (Redmond, WA).

\section{Mechanical stimulation}

We delivered mechanical stimuli (500 msec duration) to the body wall, using a solenoid-driven push rod (Guardian, Woodstock, IL) with a nylon filament $(1.6 \mathrm{~cm}$ in length and $200 \mu \mathrm{m}$ in diameter) attached to one end. Once such filaments buckle, they exert forces that are relatively independent of displacement (the Von Frey principle; see Levin et al., 1978). The solenoid was controlled with a relay circuit powered by two $9 \mathrm{~V}$ batteries in series and triggered by a Grass stimulator (Grass Instruments, Quincy, MA). We positioned the stimulus apparatus, using a micromanipulator, so that the filament tip was as close as possible to the skin without touching (within $0.5 \mathrm{~mm}$ ). During a stimulus the push rod moved $\sim 2 \mathrm{~mm}$. We calibrated the filament with a force transducer (Biocom, Culver City, CA). The mean and SD of the force produced were $21.9 \pm 0.27 \mathrm{mN}$ (five trials). In addition to this standard stimulus, one of two other nylon filaments (differing from the standard only in length, 2.0 and $0.9 \mathrm{~cm}, 10.8 \pm 0.35$ and $36.6 \pm 1.06 \mathrm{mN}$, respectively; mean $\pm \mathrm{SD}$, five trials) was used for the experiments in which two stimuli were delivered simultaneously. Each of these stimuli reliably elicited a local bend response but did not activate the high-threshold nociceptive N-cells.

\section{Measuring motor output and behavior}

We used three techniques to describe the local bend behavior. First, we used video motion analysis in preparations consisting of four intact midbody segments to observe the intact behavior; second, we measured the tension generated by body wall muscle; and third, we used electromyography (EMG). We have obtained similar results in each case, providing reasonable evidence that our results do not depend on the method of measurement.

Video motion analysis. We videotaped the local bend response in a preparation consisting of four intact segments (MS8-MS11). This was the largest number of intact segments that would not result in the continuous generation of swimming movements (J. E. Lewis, unpublished observations). Although this preparation may not allow for normal internal pressures (Wilson et al., 1996), its primary advantage is that there is normal mechanical coupling of the cylindrical body wall within the segments tested, and thus the normal behavioral response can be measured (mechanical coupling is compromised by the semi-intact preparations used for the tension and EMG measurements; see the following). One disadvantage of this preparation is that the locations for measuring response and giving stimuli are limited to an area around the dorsal midline. Another difference between this intact preparation and the semi-intact preparations involves the possibility of activating sensory neurons from segments other than the one being tested. These cells could be activated via their secondary receptive fields (Yau, 1976; Gu, 1991), resulting in an increased sensory input for a given stimulus, as compared with semi-intact preparations consisting of isolated segments. In a series of ganglion-body wall experiments, we measured the extent to which Tand P-cells were activated by a mechanical stimulus delivered to their secondary receptive fields. The mechanical stimulus used in the present study elicited no P-cell action potentials in any trial (5 animals, 10 cells, 34 trials) and in T-cells produced, at most, $10 \%$ of the number of action potentials elicited by primary field stimulation ( 4 animals, 9 cells, 27 trials). Therefore, because secondary field activation provides such a small component of the sensory input, we feel justified in comparing results from video experiments with those using EMG and tension measurements in which only one ganglion is present and, hence, no secondary field innervation.

To evoke the local bend response, we gave mechanical stimuli at four sites $\left(S=-45^{\circ},-27^{\circ}, 0^{\circ}\right.$, and $27^{\circ}$; see Fig. $1 B$ for definition of body perimeter coordinates) along the central annulus of MS10. The experiments were videotaped (Sanyo model VDC 3825) for later analysis. Figure $2 A$ shows two video frames corresponding to a single stimulus trial; the left frame is the control state (before stimulation), and the right 

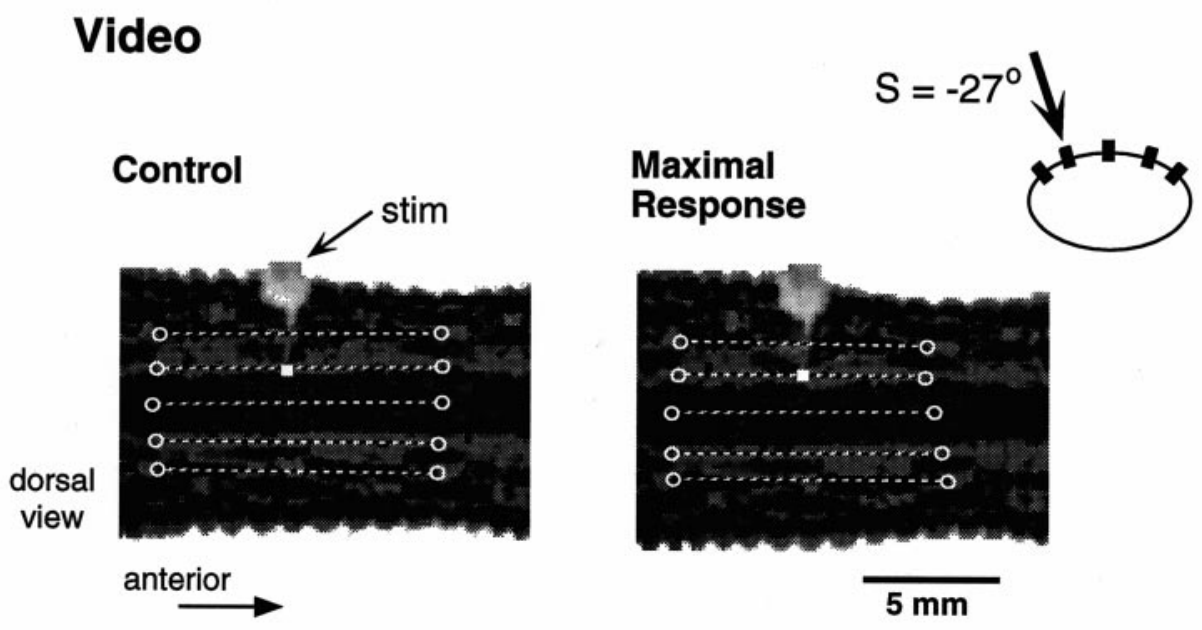

B

\section{Body wall tension}
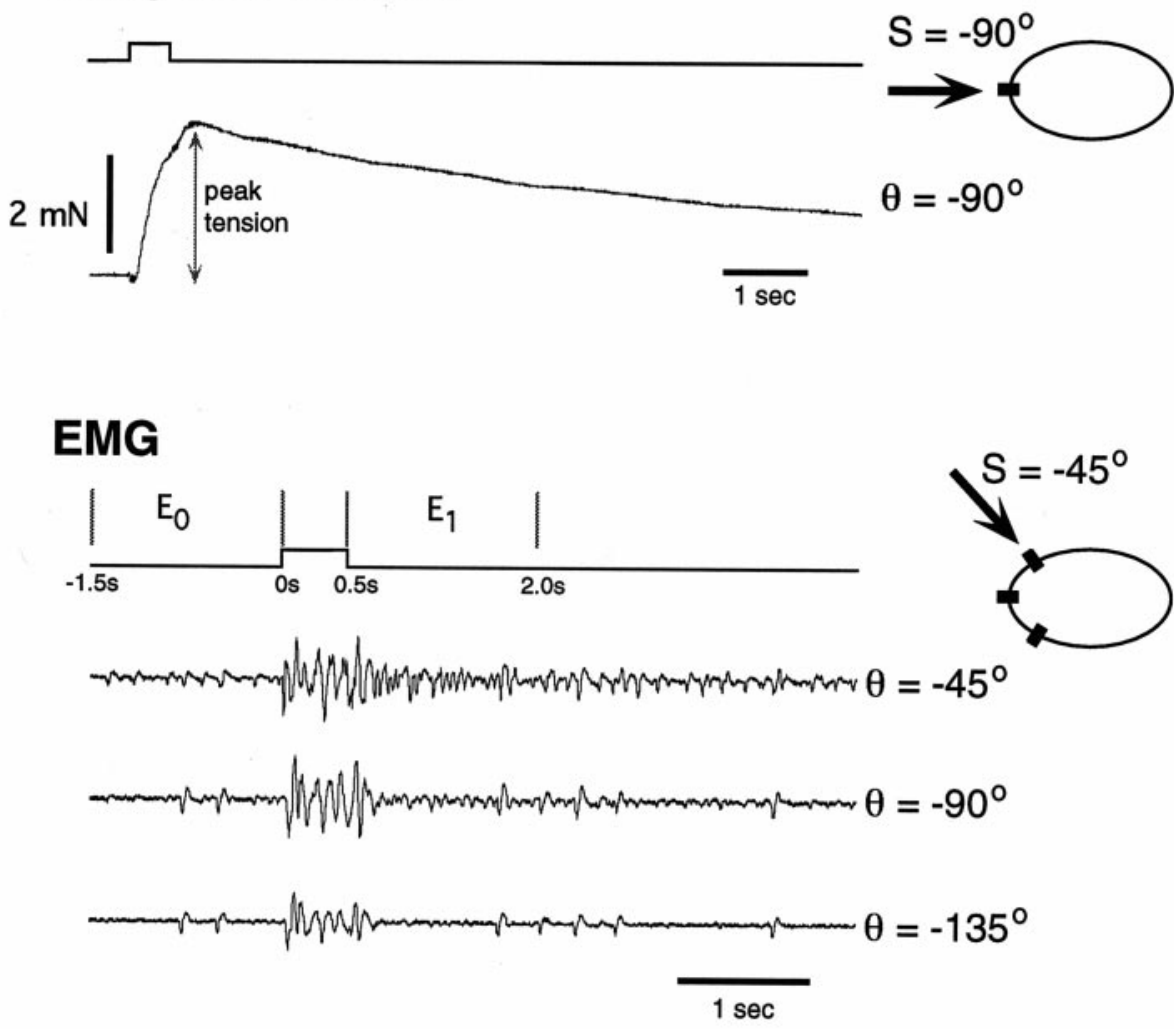

Figure 2. Three methods of measuring the local bend response. In each panel an icon representing the body perimeter shows the stimulus location $S$ (denoted by the arrow) and the measurement location $\theta$ (black rectangle). A, Video analysis. Two video frames (before and after a stimulus trial) show a dorsal view of midbody segment 10 . The stimulator is mostly out of focus in the top of the image; the stimulus location is denoted by a white square. The unfilled circles drawn on each image define the line segments used to measure the response. $B$, Body wall tension. Tension is measured at one body location for a single stimulus trial; the peak tension is indicated. $C$, Electromyographic $(E M G)$ analysis. Shown are EMG signals from three body locations for a single stimulus trial $\left(S=-45^{\circ}\right)$. Stimulus trace (top) shows the time intervals $E_{0}$ and $E_{1}$ (in seconds) during which the EMG signals were analyzed (see Materials and Methods).

frame is the maximally contracted state. We measured the local bend response as the relative longitudinal length change at five locations on the body wall perimeter $\left(\theta=-45^{\circ},-27^{\circ}, 0^{\circ}, 27^{\circ}\right.$, and $\left.45^{\circ}\right)$. The length change at each location was measured by defining a line segment connecting two prominent markings in the body wall pigment (denoted schematically by unfilled circles in Fig. $2 A$ ). In every experiment it was possible to define unambiguous markings in this way, such that the length of the line segment between two markings could be measured before and after a stimulus. In each of five animals, five stimulus trials were given every 2 min at each of the four stimulus locations (randomized block trials).

Muscle tension measurements. As in previous studies of the local bend behavior, we used the tension produced by longitudinal muscles as another indicator of local bend response (Kristan, 1982; Lockery and Kristan, 1991). This technique provides an easily quantified measure of the local bend behavior, but measuring the response at multiple locations is difficult. In ganglion-body wall preparations consisting of a single innervated segment (MS9 or MS10) from six animals, we measured muscle tension evoked by mechanical body wall stimuli (Fig. $2 B$ ). Sutures (6-0, Ethicon, Somerville, NJ) were tied to a denervated part of the body wall at either of two locations $\left(-90^{\circ}\right.$ or $\left.+90^{\circ}\right)$ and attached to force transducers (Biocom). Stimuli were given at 10 different locations every $2 \mathrm{~min}$ (randomized block trials). The response was quantified as the peak tension (subtracted from baseline) generated during a given trial. The peak tension of the local bend response occurred $\sim 1 \mathrm{sec}$ after stimulus onset. Another possible measure of the tension response is the area under the tension-time curve; this measure varies linearly with the peak tension (J. E. Lewis, unpublished observations). Peak tension measurements for a given animal were normalized to the mean value evoked for the stimulus site that resulted in the largest response.

Electromyography $(E M G)$. EMG recordings were made from the body 


\begin{tabular}{|c|c|c|c|c|c|}
\hline \multicolumn{3}{|l|}{ EMG } & \multicolumn{3}{|l|}{ Video } \\
\hline Stimulus site & A & $\theta_{\mathrm{o}}$ & Stimulus site & A & $\theta_{\mathrm{o}}$ \\
\hline $180^{\circ}$ & -0.68 & $144^{\circ}$ & $-45^{\circ}$ & -0.41 & $-72^{\circ}$ \\
\hline$-135^{\circ}$ & -0.40 & $-125^{\circ}$ & $-27^{\circ}$ & -0.29 & $-61^{\circ}$ \\
\hline$-90^{\circ}$ & -0.40 & $-115^{\circ}$ & $0^{\circ}$ & 0.18 & $-9^{\circ}$ \\
\hline$-45^{\circ}$ & -0.90 & $-34^{\circ}$ & $27^{\circ}$ & -0.27 & $58^{\circ}$ \\
\hline
\end{tabular}

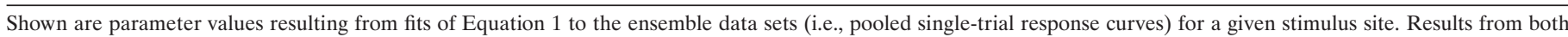
EMG (left) and video (right) measurements are shown.

wall muscle at different locations along the body perimeter, using methods described previously (Lewis, 1997). Briefly, EMG electrodes consisted of a minuten pin soldered to a flexible wire; the electrodes were insulated except for $2-3 \mathrm{~mm}$ at the tip and were inserted parallel to the longitudinal axis of the body. The reference electrode was placed in the bath, resulting in monopolar recordings. These monopolar electrodes provided EMG recordings that were similar to those obtained with other electrode configurations (e.g., bipolar, insulated silver wire alone) but were much easier to insert and thus minimized damage to the preparation. The signals were recorded with a differential amplifier (A-M Systems, Everett, WA), bandpass-filtered $(10-500 \mathrm{~Hz})$, and stored on computer for further analysis. The advantage of this technique is that it can be used to monitor the response at many locations in a semi-intact preparation while recording from neurons in the local bend network. The disadvantage is that it measures electrical activity in the muscle and does not, in a strict sense, indicate the behavioral output.

We constructed EMG tuning curves by using the following procedure. In ganglion-body wall preparations (MS10) from five animals, we mechanically stimulated the body wall at seven different sites, $S= \pm 45^{\circ}$, $\pm 90^{\circ}, \pm 135^{\circ}$, and $180^{\circ}$, and measured the EMG responses at four body wall locations, $\theta=-45^{\circ},-90^{\circ},-135^{\circ}$, and $180^{\circ}$. In a given experiment we delivered stimuli every 2 min in a randomized block trial paradigm, with 5 min between blocks. Figure $2 C$ shows EMG responses from three locations $\left(\theta=-45^{\circ},-90^{\circ}\right.$, and $\left.-135^{\circ}\right)$ for a single stimulus trial $(S=$ $-45^{\circ}$ ). In another set of EMG experiments two stimuli were delivered to different locations simultaneously. This will be referred to as the twostimuli protocol.

To quantify the change in EMG activity evoked by a stimulus, we measured the area under the full-wave rectified signal over two $1.5 \mathrm{sec}$ intervals (Fig. $2 C)$ : the control period $\left(E_{0}\right.$, beginning $1.5 \mathrm{sec}$ before stimulus onset) and the local bend period $\left[E_{1}\right.$, beginning at stimulus offset (i.e., $0.5 \mathrm{sec}$ after stimulus onset)]. Then the response was quantified by $\left(E_{1} / E_{0}-1\right)$ and referred to simply as the EMG response. We did not include the $500 \mathrm{msec}$ stimulus interval in $E_{1}$ because the EMG signal during this time is dominated by L-cell-related activity. The L-cell is a longitudinal motor neuron with an innervation field spanning one-half of the body wall (Stuart, 1970). In preliminary experiments we found that the response of the L-cell to local bend stimuli is limited mostly to the duration of stimulation. The response of cell 3 can last for many seconds after a local bend stimulus (Lockery and Kristan, 1991). In addition, L-cell activation does not appear to depend on stimulus location (J. E. Lewis, unpublished observations). Kristan (1982) observed that, for dorsal stimuli, the ventral longitudinal muscle transiently contracted before a strong ultimate relaxation response. This transient response is attributable to the L-cell. Thus, we conclude that the L-cell does not play a major role in the directionality of the local bend response, the focus of this study, so its contribution will be ignored.

\section{Tuning curves and single-trial response curves}

Tuning curves are plots of the average response of a system as a function of some stimulus parameter (Knudsen et al., 1987; Churchland and Sejnowski, 1992). We use tuning curves as one way of characterizing the local bend behavior as a function of stimulus location on the body wall perimeter. We refer to plots of EMG response (measured at a single body wall location) versus stimulus location as EMG tuning curves. Likewise, we refer to such curves for tension measurements as tension tuning curves. We were not able to construct tuning curves for video measurements because of the limited range of stimulus locations in these experiments.

Because tuning curves are constructed by measuring the response at one location while stimulating at many, they are particularly useful when technical limitations make response measurements at many locations difficult. However, because ultimately we want to characterize the sensorimotor transformation in the local bend, we also analyzed the response on a trial-by-trial basis. Therefore, we define a single-trial response curve as EMG response versus electrode location (or relative shortening vs location for video measurements). This curve describes the response profile over the body perimeter for a single trial and a single stimulus location.

\section{Estimating behavioral accuracy}

We define the behavioral error as the difference between stimulus location and bend direction. To determine bend direction, we performed the following analysis on the single-trial response curves (EMG and video data). The single-trial response curves were bell-shaped with a central maximum (see Fig. 5). We used the body wall location where the maximum response occurs, $\theta_{\max }$, as an indicator of bend direction. To estimate $\theta_{\max }$ at a finer resolution than our measurement resolution, we used the following interpolation procedure.

The single-trial response curves were normalized so that the maximum value in each was equal to one. Data from all trials were pooled for each stimulus site, resulting in eight ensemble data sets (four each for both video and EMG data). We chose a cosine function $f(\theta)$, with two free parameters, $A$ and $\theta_{\mathrm{o}}$, to fit the data:

$$
f(\theta)=\frac{R_{\mathrm{o}}}{1-A}\left\{\cos \left(\theta-\theta_{\mathrm{o}}\right)-A\right\},
$$

where $R_{\mathrm{o}}=1$ defines the peak amplitude of the response. The values for the parameters $A$ and $\theta_{\mathrm{o}}$ were determined by a regression analysis on the ensemble data sets (thus providing a population estimate of these parameters). Because the data at different measurement sites did not always show uniform variance, we used a two-step weighted least-squares method (Chatterjee and Price, 1991). The first step consisted of an ordinary least-squares (OLS) fit to the data. The second step involved a least-squares fit weighted by the reciprocal of the variance of the OLS residuals. The peak locations of the individual trials were determined by fitting the single-trial response curves to the same function (Eq. 1) but with $A$ fixed at the values determined by the ensemble analysis (Table 1 ) so that $\theta_{\mathrm{o}}$ was the only free parameter in this step. Again, a weighted least-squares method was used with identical weights as for the ensemble fits. The value of the parameter $\theta_{\mathrm{o}}$ from these fits was taken as the value for $\theta_{\max }$, the body wall location of peak response. The quality of fit varied for the single-trial response curves. Any trial in which the fitted curve accounted for $<65 \%$ of the variance in the data was not considered in further analyses. This selection criterion resulted in acceptance of 94 and $65 \%$ of the trials for the video and EMG experiments, respectively. The high acceptance rate for the video trials confirms that the cosine function is appropriate for describing the single-trial response curves at the behavioral level. The relatively low acceptance rate for the EMG trials could be attributable to a more variable signal at the level of electrical activity in the muscle (that is subsequently smoothed by the biomechanics) or to more experimental error involved in recording this signal. For the two-stimuli protocol experiments, the single-trial response curves were not normalized, and $R_{\mathrm{o}}$ in Equation 1 was also a free parameter.

\section{Statistical analysis}

The data relating to stimulus location and body perimeter location are periodic and thus were analyzed as circular data (Batschelet, 1981). The data in Figure 6 are compared with the predictions for each behavioral 
strategy by calculating the residuals (i.e., the angular distance between a data point and the strategy prediction for the corresponding stimulus site). Because the predictions for the Categorical model are discontinuous at four stimulus locations, the residuals at these locations were calculated by a random choice of one or the other predicted value across the discontinuity. Then the squared residuals for the different strategies were compared with a $\chi^{2}$ test. Variance (or angular dispersion) in the peak response location between different stimulus sites was compared with the Wilcoxon-MannWhitney $U$ test for circular data (Batschelet, 1981).

\section{RESULTS}

\section{Tuning curves}

Figure 3 shows EMG tuning curves for four measurement locations, $\theta$. In general, the EMG response is maximal for a stimulus site equal to the EMG measurement location and decreases for stimulus sites farther from this location. An illustration of this trend is that for each measurement location a cosine curve can account for $>85 \%$ of the variance in the mean data (Fig. $3 A-D$ ). If the peak of the cosine is set equal to the measurement location, the curve can still account for $>80 \%$ of the variance in each case. This trend is evident in individual animals as well, where on average $82 \%$ of the variance can be accounted for by a cosine curve. Although the cosine model is quite simplistic, it appears to capture the main features of the EMG tuning curve data. However, the tuning curves for $\theta=-90^{\circ}$ and $\theta=-135^{\circ}$ appear quite similar; in both cases the peak response in a given animal occurred at either $S=-90^{\circ}$ or $S=-135^{\circ}$. Such ambiguities can result when average responses are investigated, and we will address this issue in our investigation of individual trials.

EMG activity reflects the electrical activity of the muscle and does not necessarily reflect the resulting movement. Ideally, we could measure tuning curves while videotaping the actual movements, but because of technical limitations we were unable to do so (see Materials and Methods). Instead, we compared an EMG tuning curve with one constructed that used the evoked body wall tension (Fig. 4). The data are similar in both cases, although the tension tuning curve appears to be slightly more narrow, with very little response for contralateral stimuli. The best fit cosine curve can account for $92 \%$ of the variance in the mean tension data; the same curve (i.e., same parameter values) also can account for $90 \%$ of the variance in the mean EMG data.

\section{Behavioral accuracy: single-trial analysis}

In both EMG and video experiments we measured the single-trial response curves and used an interpolation procedure (see Materials and Methods) to determine $\theta_{\max }$, the body location where the maximal response occurs. Single-trial response data were pooled for all trials at a given stimulus location (EMG and video data were analyzed separately). Figure $5 A$ shows an example of such an ensemble data set for video data $\left(S=0^{\circ}\right)$, along with the corresponding regression curve (Eq. $\left.1, R_{\mathrm{o}}=1\right)$ used to obtain a population estimate of the parameter, $A$. The resulting values for $A$ are shown in Table 1 . Then the single-trial response curves were fit to the same function (Eq. 1) but with $A$ fixed at the ensemble values (Table 1). The only free parameter in this step was $\theta_{\mathrm{o}}$, for which the resulting value was taken as $\theta_{\text {max }}$. Figure $5 B$ shows two single-trial response curves with their corresponding curve fits. These examples provide an idea of the range in curve fit quality; one of the best fits is shown by the solid line (accounting for $99 \%$ of the variance in the data), whereas one of the worst fits is shown by the dotted line (accounting for only $42 \%$ of the variance and thus it was not considered for further analyses; see Materials and Methods).

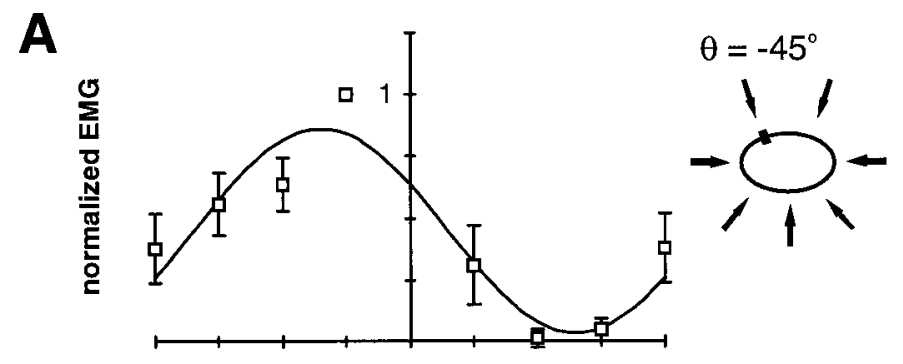

B
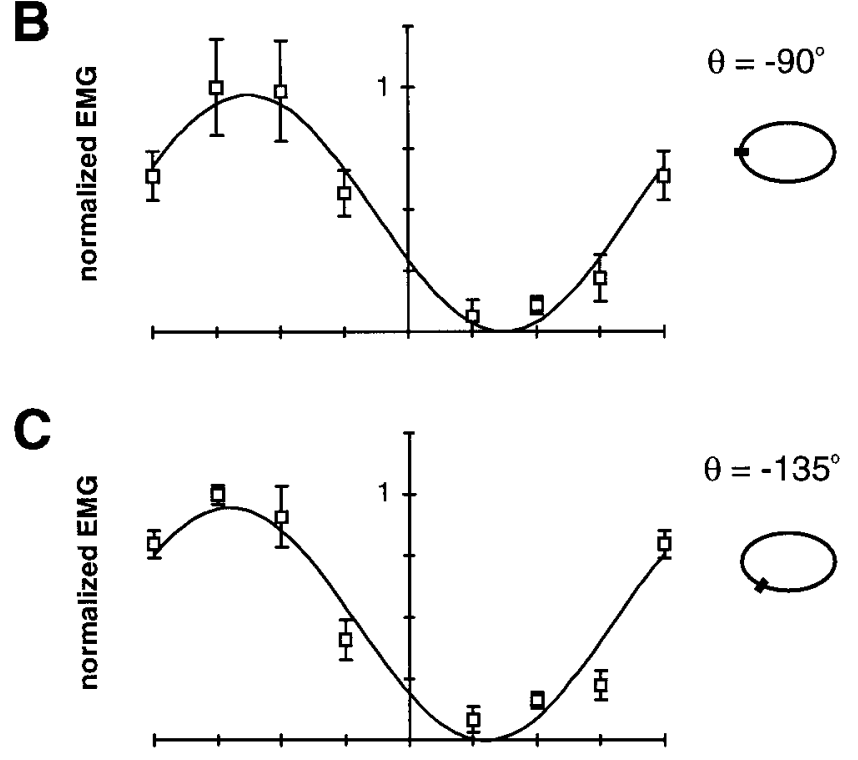

D

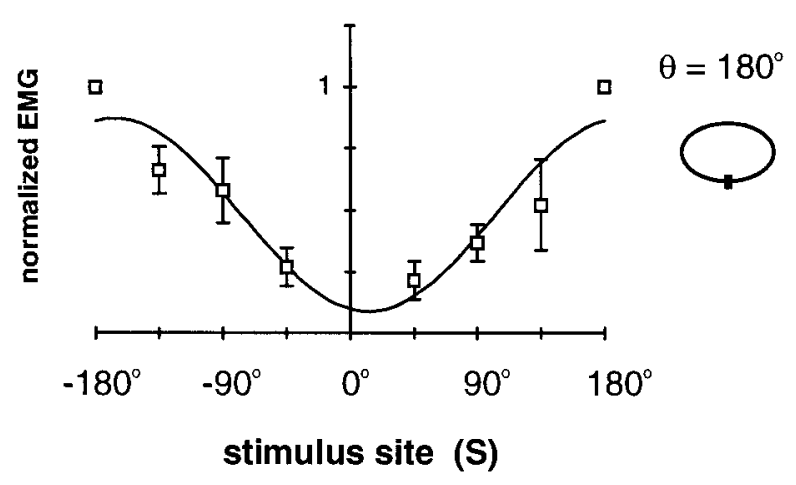

Figure 3. EMG tuning curves. Normalized EMG response at four body locations is plotted against stimulus location. In $A$, the body perimeter icon illustrates the stimulus locations (arrows), and in all panels this icon shows the location of EMG measurement, $\theta$ (small filled rectangles). $A, \theta$ $=-45^{\circ} ; B, \theta=-90^{\circ} ; C, \theta=-135^{\circ} ; D, \theta=180^{\circ}$. Data points show the mean \pm SEM for five animals. Also shown is the cosine curve, $b_{0}+b_{1} \cos$ $\left(S-S_{\mathrm{o}}\right)$, which best fits the mean data: $A, b_{0}=0.45, b_{1}=0.41, S_{\mathrm{o}}=-63^{\circ}$; $R^{2}=0.89 . B, b_{0}=0.48, b_{1}=0.48, S_{\mathrm{o}}=-113^{\circ} ; R^{2}=0.97 . C, b_{0}=0.47$, $b_{1}=0.47, S_{\mathrm{o}}=-126^{\circ} ; R^{2}=0.92 . D, b_{0}=0.48, b_{1}=0.39, S_{\mathrm{o}}=-167^{\circ}$; $R^{2}=0.85$.

Figure 6 shows a scatter plot for $\theta_{\max }$ as a function of stimulus site. On average, the local bend responses are centered close to the stimulus site (circular correlation, $r=0.89$; $p<0.001$, Rayleigh test). The root mean-squared (RMS) difference among peak location, $\theta_{\max }$, and stimulus site was $28^{\circ}$. In other words, on average, the local bend network produces a behavioral output 


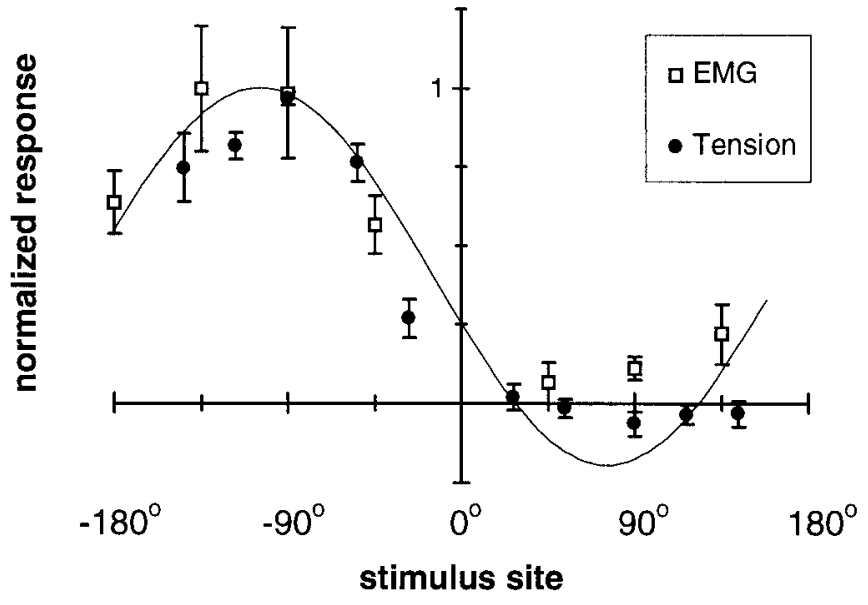

Figure 4. Tension tuning curve. Filled circles show the normalized peak tension measured at $\theta=-90^{\circ}$ plotted against stimulus location (each point is the mean \pm SEM for six animals). Also plotted are the EMG data (unfilled squares) from Figure $3 B$ for comparison. The cosine curve is a best fit to the peak tension data $\left(b_{0}=0.40, b_{1}=0.60, S_{\mathrm{o}}=-104^{\circ} ; R^{2}=\right.$ 0.92; see Fig. 3 legend).

that is directed within $8 \%$ of the stimulus location, corresponding to an accuracy of $\sim 1.6 \mathrm{~mm}$ (the perimeter of the leech body wall is $\sim 2 \mathrm{~cm}$ in length). This suggests that the local bend behavior involves a strategy in which the response varies continuously with stimulus location (Continuous strategy). However, in the following we show that the data are also consistent with a specific form of Categorical strategy.

\section{Comparing Continuous and Categorical strategies: single-trial analysis}

The Categorical strategy we considered involves four distinct forms of the behavior (i.e., bend direction), distinguished by the quadrant of the body wall perimeter where the stimulus is given. The quadrants are defined by transition regions at $\theta=0^{\circ}, 90^{\circ}$, $180^{\circ}$, and $-90^{\circ}$. These quadrants correspond approximately to the innervation fields of the four $\mathrm{P}$ sensory neurons (Nicholls and Baylor, 1968; Lewis, 1997), providing a plausible physiological mechanism for this Categorical strategy (i.e., a winner-take-all mechanism mediated by the P-cells). This Categorical strategy makes specific predictions about the form of the curve shown in Figure 6: there are four different peak response locations for all possible stimulus sites, such that the curve is piecewise constant with four steps (gray line). The corresponding prediction for the Continuous strategy is the identity line (i.e., where the peak location is equal to the stimulus site). We evaluated the predicted peak location for both strategies with the data by comparing the distributions of the squared residuals; the Continuous strategy was a significantly better fit to the data ( $p=0.045 ; \chi^{2}$ test). Even so, the Categorical strategy accounted for almost $80 \%$ of the variance in the data with an RMS residual of $36^{\circ}$, whereas the Continuous strategy accounted for $83 \%$ of the variance (RMS residual $=28^{\circ}$ ).

Another aspect in which the predictions of the two strategies differ is the variance (i.e., angular dispersion) of the behavioral response. The Continuous strategy predicts that response variance should not vary with stimulus location. The Categorical strategy predicts that stimuli given at the transitions between quadrants (i.e., $S=0^{\circ}, 90^{\circ}$, $180^{\circ}$, and $-90^{\circ}$ ) should produce responses that either are distributed bimodally or are much more
A

\section{Ensemble data set}

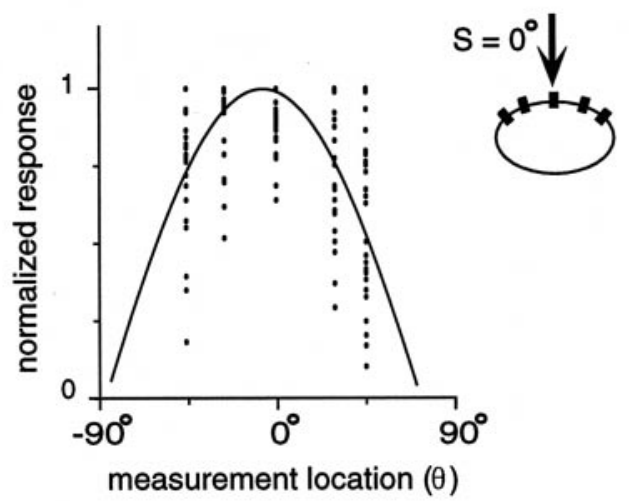

B

\section{Single-trial response curves}

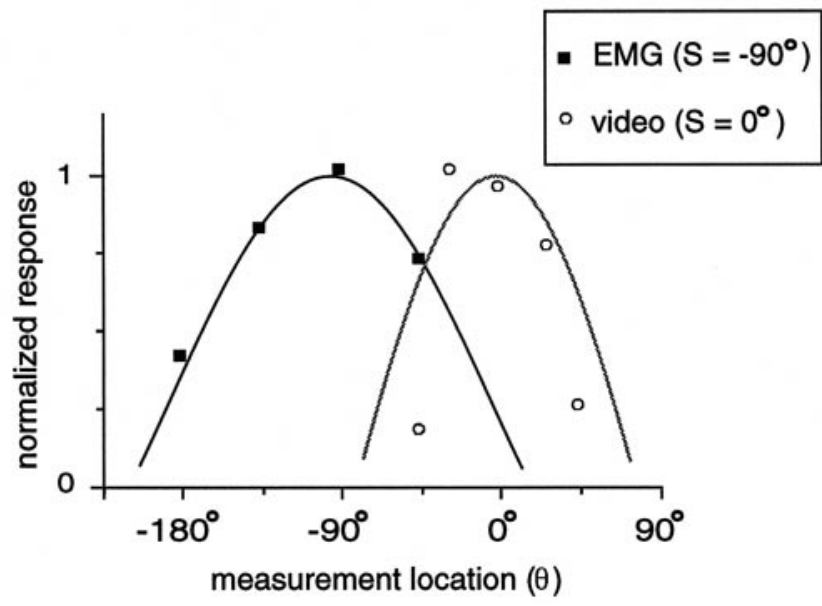

Figure 5. Measuring single-trial response. $A$, Ensemble data set. Plot shows normalized response (video analysis) versus measurement location on the body wall. A single trial consists of one measurement from each of the measurement locations, where the maximal response is set to one. The solid curve shows the cosine function that best fits the ensemble data (see Materials and Methods). B, Single-trial response curves. Shown are a single stimulus trial for EMG (solid squares; $S=-90^{\circ}$ ) and video (unfilled circles; $S=0^{\circ}$ ) measurements, along with the respective curve fits. These trials provide examples of the best and worst fits (see Materials and Methods).

variable (increased variance). There is no evidence of a bimodal distribution for the responses at any of the stimulus sites. However, to test the possibility that the variance was greater in the presumptive transition regions, we calculated the angular distance between each data point and the corresponding mean and then pooled the data into two groups: transition zones (i.e., $S=0^{\circ}$, $-90^{\circ}$, and $180^{\circ}$ ) and nontransition zones, where the two strategy predictions are the same $\left(S=-45^{\circ}\right.$ and $\left.-135^{\circ}\right)$. No significant difference was observed between these two groups $(p=0.12)$.

Considering these results, it appears that the Continuous strategy best supports our data. In both comparisons the difference between the two strategies was fairly small, so we have considered two additional levels of analysis, a simple model and a two-stimuli protocol, to distinguish between the Continuous and Categorical strategies. 


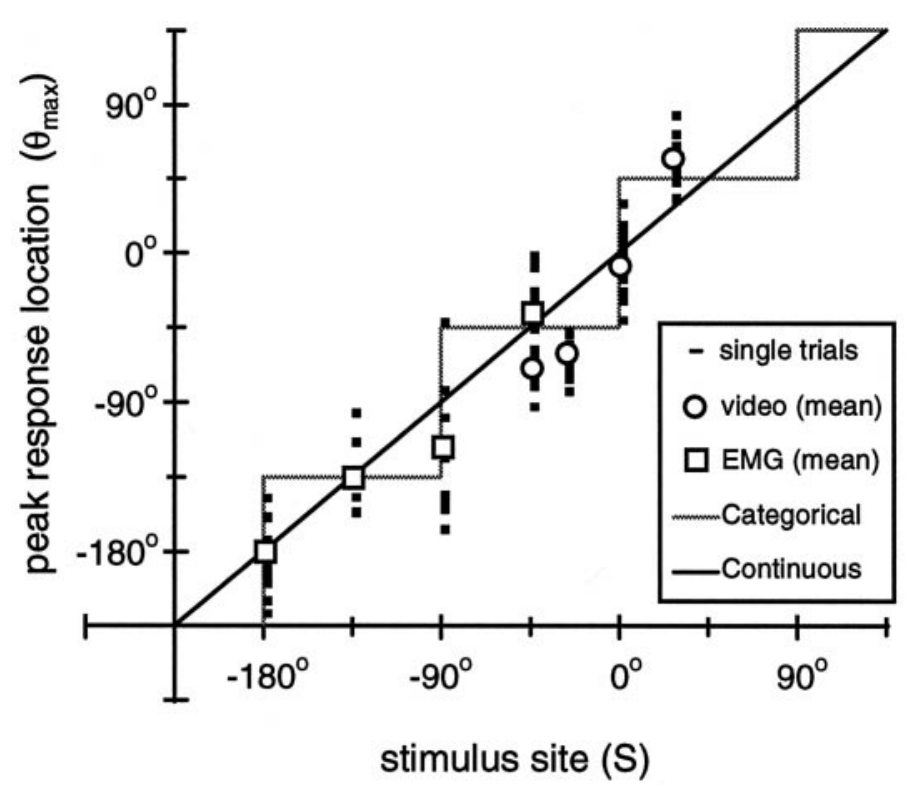

Figure 6. Single-trial peak response location. Shown is the relationship between peak response location $\left(\theta_{\max }\right)$ and stimulus site $(S)$. Mean (unfilled symbols) and single-trial data (dashes) are shown for both EMG and video measurement techniques. Note that the single-trial responses overlap, and thus the dashes appear as squares. The identity line, which is drawn for reference, also represents the predicted responses for the Continuous strategy. The prediction for the Categorical strategy is denoted by the shaded gray line.

\section{Predicting motor output by using a simple model}

In this section we outline a simple approach for predicting the tuning curves of the motor neurons that innervate the longitudinal body wall muscle. These predictions are made in the context of both the Continuous and Categorical strategies with the intent of localizing differences between the two strategies at the level of motor output. We then can compare these predictions with what is known about the activation of motor neurons during a local bend.

\section{The idealized response}

So far, we have discussed the local bend response in terms of actual movements (video), muscle tension, and EMG, but in the following we consider a generalized local bend response. The local bend response is a function of stimulus location, $S$, and the location on the body perimeter where the response is measured, $\theta$. We define an idealized response function, $R(S, \theta)$, and then fit the motor output model to this function. The form that the function $R(S, \theta)$ takes is different for the Categorical and Continuous strategies. The EMG tuning curves in Figure 3 are well described by cosine curves, suggesting that $R(S, \theta)$ should be continuous in $S$ for both strategies (this is a constraint imposed by the data). So we consider $R(S, \theta)$ to be of the form:

$$
R(S, \theta)=0.5\left\{1+\cos \left[S-S_{\max }(\theta)\right]\right\},
$$

where $S_{\max }(\theta)$ defines the peak of the tuning curve and differs for the two behavioral strategies. For the Continuous strategy, $S_{\max }(\theta)=\theta$, so that the peak of the tuning curve occurs at the body wall measurement location, $\theta$. For the Categorical strategy the function $S_{\max }(\theta)$ is piecewise constant (Eq. 3) so that the tuning curve peaks occur at one of four locations, depending on the body location:

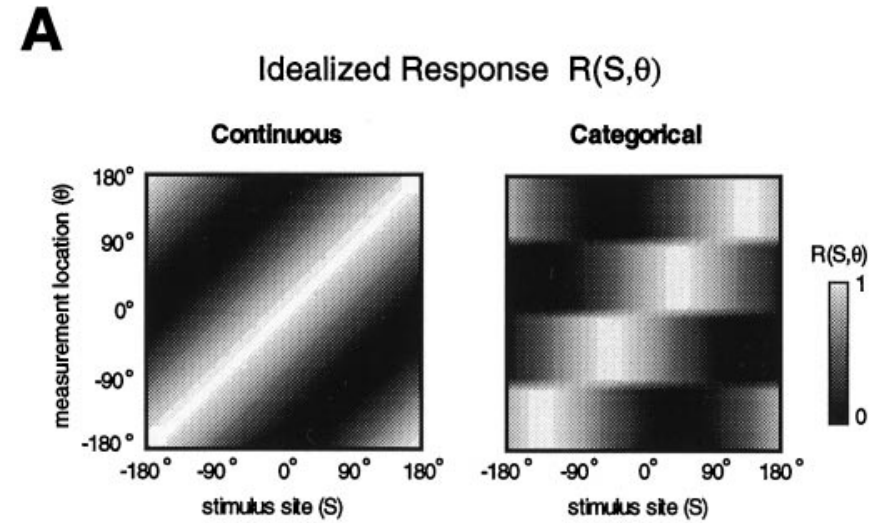

B

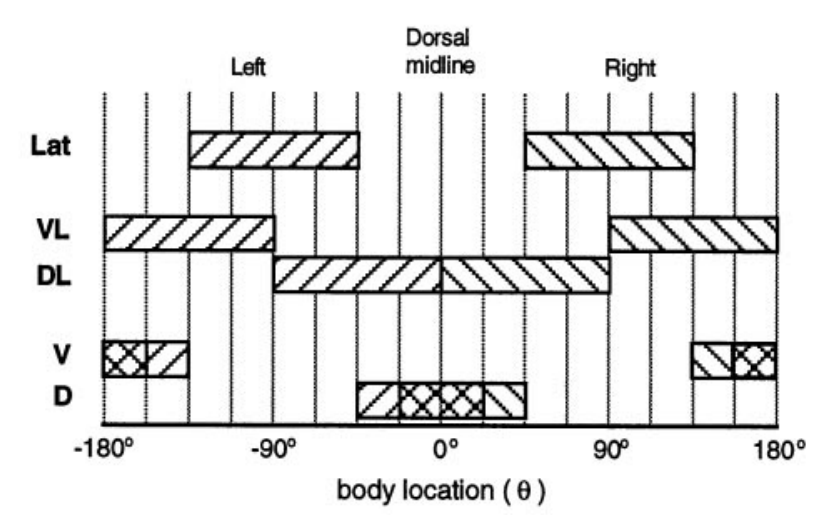

Figure 7. The basis for a model of motor output. $A$, Idealized response $R(S, \theta)$. The predicted responses are shown for each behavioral strategy as a function of stimulus site $(S)$ and body wall measurement location $(\theta)$. The response magnitude is given in gray scale, with white being the largest response. A tuning curve is a horizontal line slice through this plot (i.e., responses at a single location to stimuli at all locations), and a single-trial response curve is a vertical line slice (i.e., responses at all locations for a stimulus at one location). $B$, Body wall innervation. Shown are innervation fields for the five classes of longitudinal motor neurons considered in the model. Right and left homologs for a given class are distinguished by different diagonal filled patterns. Overlapping fields within the $D$ and $V$ classes are shown by cross-hatching. The discretization of the body perimeter into 16 bins is indicated by the vertical lines.

$$
S_{\max }(\theta)=\begin{array}{rcc}
45^{\circ} & \left(0^{\circ}<\theta \leq 90^{\circ}\right) \\
135^{\circ} & \left(90^{\circ}<\theta \leq 180^{\circ}\right) \\
-135^{\circ} & \text { if } \quad\left(-180^{\circ}<\theta \leq-90^{\circ}\right) \\
-45^{\circ} & & \left(-90^{\circ}<\theta \leq 0^{\circ}\right) .
\end{array}
$$

The response functions $R(S, \theta)$ for each strategy are shown in Figure $7 A$. The body location and stimulus site are represented on the vertical and horizontal axes, respectively; the magnitude of the response is represented in gray scale (a large response is white, and a weak response is black). A tuning curve at a given body location, $\theta^{*}$, is a horizontal slice through these plots intersecting $\theta^{*}$. Alternatively, a single-trial response curve is analogous to a vertical slice through these plots.

\section{Anatomical constraints}

There are five distinct classes of excitatory longitudinal motor neurons, named for the area of longitudinal muscle that they innervate (Stuart, 1970; Mason and Kristan, 1982; J. E. Lewis, 


\section{Motor output model}

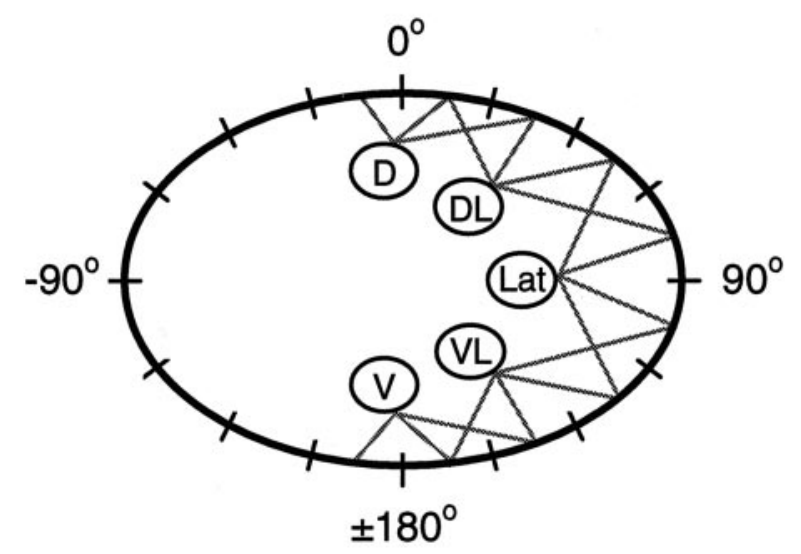

Stimulus $S$

Figure 8. Motor output model. Given is a schematic illustration of the model used to predict motor neuron tuning. The body perimeter is shown in conventional coordinates; the 16 bins are shown by the dashes. The motor neurons innervating the right body wall are shown, with the gray lines indicating connections to a particular bin (innervation matrix). In the box, a schematic flow of the model is provided. A stimulus at a location $S$ results in activation of the different motor neurons, given by $M(S)$. This motor neuron activity is passed through a threshold function. The model response $R_{\mathrm{m}}$ in a particular bin is determined by the summed activity of all the motor neurons innervating that bin (given by the innervation matrix, $W$ ).

unpublished observations): dorsal (D), ventral (V), dorsolateral (DL), ventrolateral (VL), and lateral (Lat). Figure $7 B$ shows the approximate innervation fields for each of these classes. By dividing the body perimeter equally into 16 bins (see divisions in Figs. $7 B, 8$ ), we can define an innervation matrix, $W$, where an element $w_{\mathrm{ij}}=1$ if motor neuron $j$ innervates the body wall at bin $i$, and $w_{\mathrm{ij}}=0$ otherwise. The identified motor neurons within each class are cells 3 and 4 for D and V, respectively, cell 106 for Lat, cells 5, 7, and 107 for DL, and cells 8 and 108 for VL. These motor neuron classes are bilaterally symmetric, so each cell has a contralateral homolog. An additional motor neuron in the Lat class is the L-cell, but we do not consider it for the present model because its activity is transient and its innervation field (entire hemi-segment) is not compatible with either of the behavioral strategies most likely for the local bend (see Materials and Methods).

\section{Motor output model}

We assume a simple yet plausible model for relating the activity of a set of motor neurons, $M_{\mathrm{j}}(S)(j=1, \ldots, 10)$, and the response they produce (Eq. 4). The motor neuron activity is passed through a threshold function, such that only activity above zero has an effect on the motor output response. The model response $R_{\mathrm{m}}$ for a stimulus site $S_{\mathrm{k}}$ and a body wall location, $\theta_{\mathrm{i}}$, is the linear sum of the thresholded activity of all the motor neurons that innervate $\theta_{\mathrm{i}}$ (given by the innervation matrix, $W$; see Fig. 8).

$$
R_{\mathrm{m}}\left(S_{\mathrm{k}}, \theta_{\mathrm{i}}\right)=\sum_{j=1}^{10} w_{\mathrm{ij}} M_{\mathrm{j}}\left(S_{\mathrm{k}}\right) H\left(M_{\mathrm{j}}\left(S_{\mathrm{k}}\right)\right),
$$

where $i, k=(1, \ldots, 16)$ and $H$ is defined by $H(x)=0$ if $x<0$ and $H(x)=1$ if $x \geq 0$ and acts as the threshold function. The model is shown schematically in Figure 8.

\section{Calculating the motor neuron tuning curves}

The goal of this approach is to determine the functions, $M_{\mathrm{j}}(S)$, which are the motor neuron tuning curves. We do this by finding the $M_{\mathrm{j}}(S)$ that minimize the squared difference (least squares) between $R$ and $R_{\mathrm{m}}$ over all stimulus sites, $S_{\mathrm{k}}(k=1, \ldots, 16)$, and body wall locations, $\theta_{\mathrm{i}}(i=1, \ldots, 16)$. In principle, the tuning curve for a given motor neuron can be any function of $S$, with the only constraint that it is periodic with period equal to $360^{\circ}$. To constrain our predictions, we assume cosine tuning for the motor neurons, such that the $M_{\mathrm{j}}(S)$ are of the form shown in Equation 5:

$$
M_{\mathrm{j}}(S)=A_{\mathrm{j}}+B_{\mathrm{j}} \cos \left(S-S_{\mathrm{j}}^{*}\right) .
$$

This is based on the observation that at least two motor neurons, cells 3 and 4, exhibit cosine-like tuning (Lockery and Kristan, 1990a; Kristan et al., 1995). We also assume right-left and dorsal-ventral symmetry, such that the parameters $A_{\mathrm{j}}$ and $B_{\mathrm{j}}$ are equal for $\mathrm{D}$ and $\mathrm{V}$ and are also equal for DL and VL. This symmetry constraint was implemented for practical reasons, but it was not necessary because the symmetry is intrinsic to the innervation matrix, $W$. With these constraints the minimization procedure is reduced to solving for the $A_{\mathrm{j}}, B_{\mathrm{j}}$, and $S_{\mathrm{j}}{ }^{*}$ for only three motor neurons, D, DL, and Lat, with the remainder of the motor neurons specified by the results of these three. We perform the minimization for the Continuous and Categorical strategies by changing the form of the idealized response, $R$ [i.e., the choice of $S_{\max }(\theta)$ in Eq. 2]. This gives a predicted set of tuning curves for both behavioral strategies. These tuning curves reflect the effective motor neuron activation because our model does not distinguish between the absolute level of activity of a motor neuron and its relative efficacy in activating the muscle, nor does it distinguish the effects of the inhibitory motor neurons. With the use of the previous experimental data (Mason and Kristan, 1982), a more detailed model could account for such differences between motor neurons. However, because we were investigating the qualitative features of motor output, our simple model was sufficient.

\section{The model predictions}

Figure 9 shows the predicted tuning curves of the motor neurons innervating the right body wall for both behavioral strategies. The best solution for the Continuous strategy $(\mathrm{RMS}$ residual $=0.08)$ involves activation of all motor neurons, with the peaks of their tuning curves distributed at six different stimulus locations. The DL, VL, and Lat neurons are activated to a greater extent than either the $\mathrm{D}$ or $\mathrm{V}$ neurons. The DL motor neurons produce approximately twice the peak muscle tension as the D motor neuron (Mason and Kristan, 1982). Our results suggest that this difference in efficacy may be required for a continuously varying behavioral response. However, for the Categorical strategy the D, $\mathrm{V}$, and Lat motor neurons are not activated at all: the best solution in this case (near perfect with RMS residual $\cong 0$ ) involves the activation of only the DL and VL motor neurons. This is because the single-quadrant innervation fields of these motor neurons correspond directly to the four distinct regions of the Categorical strategy. The differential activation of any other 
A

\section{Continuous}

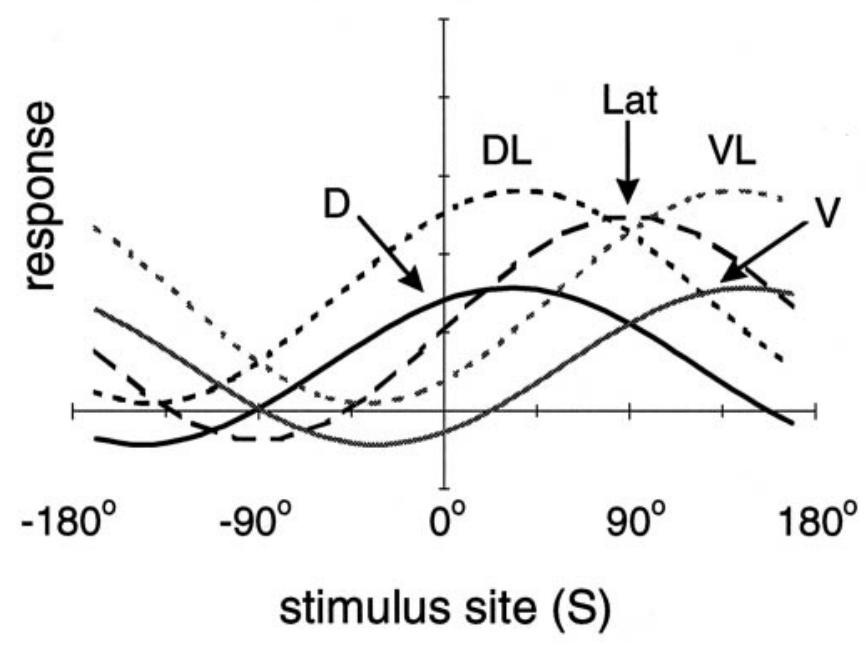

B

\section{Categorical}

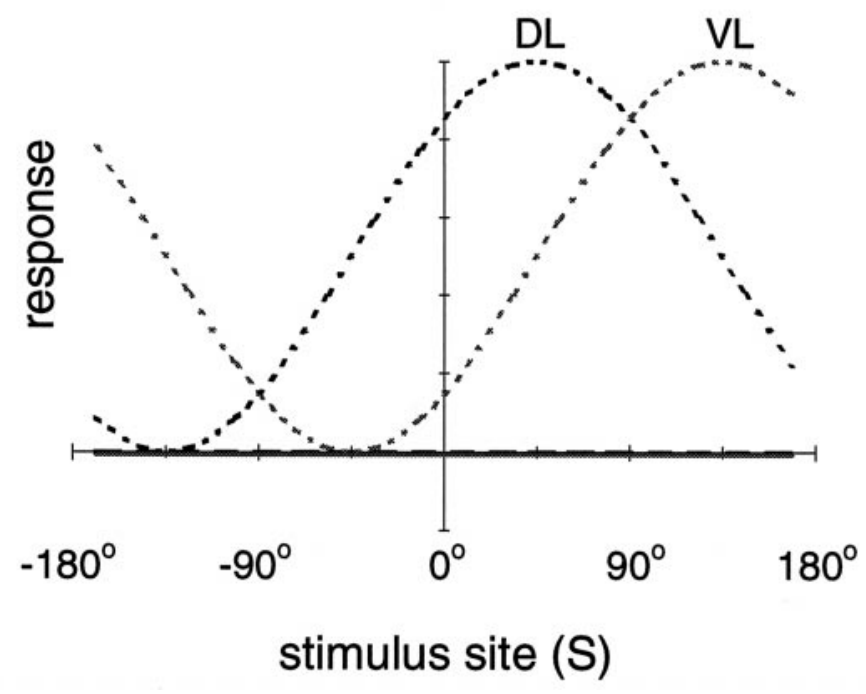

Figure 9. Predicted motor neuron tuning curves. Model tuning curves for each of the five classes of motor neurons (right side only) are shown for both behavioral strategies, Continuous $(A)$ and Categorical $(B)$. For the Categorical strategy $(B)$, the $D, V$, and Lat motor neurons do not respond at all (i.e., their tuning curves are flat).

motor neurons within these regions would change the response within the region and thus would not satisfy the Categorical strategy. These results suggest that activation of the D, V, and Lat motor neurons is incompatible with the Categorical strategy. However, the $\mathrm{D}$ and $\mathrm{V}$ neurons are known to be activated by a local bend stimulus (Lockery and Kristan, 1990a), providing evidence against the Categorical strategy.

\section{Distinguishing between Continuous and Categorical strategies: two-stimuli protocol}

A final test of the Continuous and Categorical strategies was to use a two-stimuli experimental protocol. Such experiments can distinguish between the two strategies because, in the Categorical strategy considered here, only one form of the behavior can be expressed for a given range of stimuli. This winner-take-all nature predicts that when two stimuli are given simultaneously, a single form of the behavior, corresponding to one or the other stimuli, will be expressed. Consider the stimuli, S1 and S2, that alone produce different behaviors centered at $\theta 1$ and $\theta 2$, respectively. If S1 and S2 are given simultaneously, the Categorical strategy predicts that the behavior will be centered at either $\theta 1$ or $\theta 2$. If the magnitude of S1 is greater than that of S2, the behavior centered at $\theta 1$ would be more likely. Alternatively, the Continuous strategy predicts that the resulting behavior will be centered at some intermediate location, $\theta 3$.

We performed these experiments in ganglion-body wall preparations while measuring EMG responses at three locations $(\theta=$ $-90^{\circ}, 180^{\circ}$, and $90^{\circ}$ ). Two stimuli were delivered, first individually and then simultaneously, at locations $\left(\mathrm{S} 1=-135^{\circ}\right.$ and $\mathrm{S} 2=$ $135^{\circ}$ ). The stimulus S1 was of greater magnitude than S2 (see Materials and Methods) so that one behavioral response would dominate in a winner-take-all scenario. Figure 10, $A$ and $B$, shows the results of one experiment. Each vector represents the location and amplitude of the peak response for a given stimulus trial. On average, the peak responses to $\mathrm{S} 1$ are of greater amplitude than those of S2 and are located at $\theta=-130^{\circ}$, as compared with $\theta=$ $101^{\circ}$ for S2 (Fig. 10B). Also shown are the responses to both stimuli delivered simultaneously $(\mathrm{S} 1+\mathrm{S} 2)$; on average, these responses are centered at a location intermediate to the responses to each individual stimulus. Indeed, the average peak response location for S1 $+\mathrm{S} 2$ is well described by the average of the two individual responses (i.e., vector sum). There was no evidence of a bimodal distribution of responses, which also could result in the observed average response. No simple relationship was observed between the amplitude of the response caused by S1 $+\mathrm{S} 2$ and that predicted by a vector sum. Figure $10 C$ shows the predicted peak location for both strategies versus actual peak location. The prediction for the Continuous strategy is given by the vector sum of the responses to $\mathrm{S} 1$ and $\mathrm{S} 2$, and the prediction for the Categorical strategy is the response to $\mathrm{S} 1$ alone. Points that fall on the identity line in this plot correspond to perfect predictions. In all experiments the average responses were better described by the Continuous strategy. This last piece of evidence, along with those described in previous sections, indicates that the local bend behavior can be described best by a strategy in which the response varies continuously with stimulus location.

\section{DISCUSSION}

The local bend of the medicinal leech is a directed behavior elicited by a touch to the body wall. The present paper quantified the relationship between touch location and directed behavioral output. We used a controlled mechanical stimulus to activate the mechanosensory neurons, whereas previous studies of this behavior used direct electrical stimulation of mechanosensory neurons to mimic a touch. By activating these neurons in a natural way, we allow the possibility that a realistic spike train contributes to stimulus representation and behavioral output. We also developed methods for measuring behavioral output on a trial-by-trial basis, allowing the behavior to be quantified in terms of how accurately it corresponds to stimulus location.

\section{Behavioral accuracy}

The local bend behavioral output is directed within $8 \%$ of the stimulus location, on average. Recently, the cricket escape re- 


\section{A single trials}

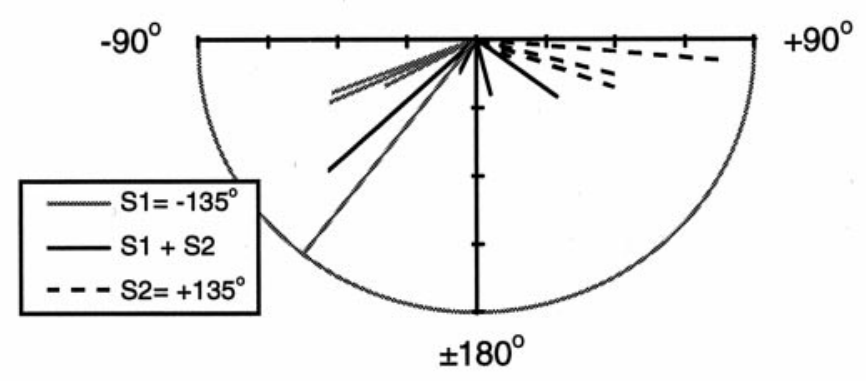

B

\section{mean responses}

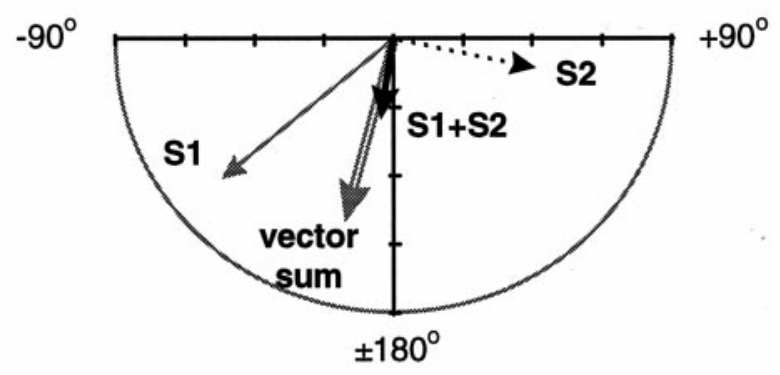

C

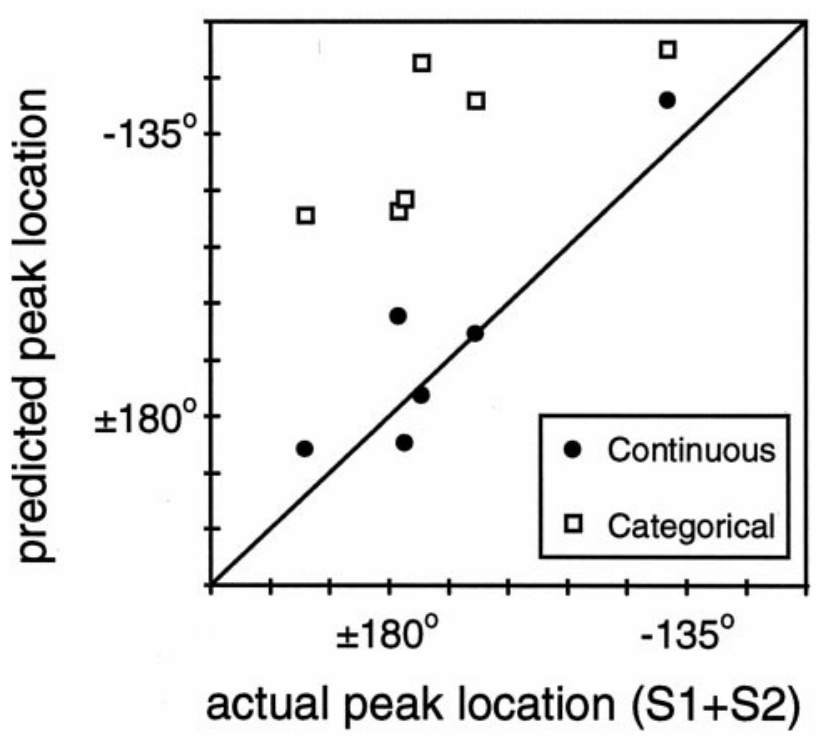

Figure 10. Two-stimuli protocol. $A$, Single-trial responses for one experiment. The directed lines are vectors for which the magnitude and direction are given by the location and amplitude of the local bend EMG response to a single stimulus trial. Three different stimuli were given: $S 1$ at location $-135^{\circ}$ ( gray lines), $S 2$ at location $135^{\circ}$ (dotted lines), and both $S 1$ and $S 2$ together at their respective locations (solid black lines). Note that the magnitude of the largest response to $S 1$ was slightly truncated for clarity (see the mean value in the panel below). $B$, Mean values for the responses in $A$ : $S 1$, gray arrow; $S 2$, dotted arrow; $S 1+S 2$, thick black arrow. Also shown is the vector sum of the mean responses to $S 1$ and $S 2$ (unfilled sponse to a directed wind source has been quantified in a way that allows the estimation of a behavioral error (Tauber and Camhi, 1995). From these data [Tauber and Camhi (1995), their Fig. 4] we estimated the average behavioral error for an escape turn to be $\sim 20 \%$. For two other variants of the response, the turn and jump and the jump, the behavioral error was closer to $10 \%$. Sound localization in barn owls is an example of a directed behavior that is much more accurate. The form of this behavior most relevant to the present context involves the open-loop paradigm. In this situation the auditory stimulus is terminated before a head turn is initiated. The sound localization error can be $<1 \%$ for frontal stimuli (sound source $<30^{\circ}$ to the right or left of center) and increases to $\sim 3 \%$ for a stimulus given at $70^{\circ}$ (Knudsen et al., 1979).

To assess the accuracy of information processing by a neuronal network, we think that it is necessary to consider the accuracy of stimulus representation by primary sensory neurons in the pathway. To date, it has not been technically possible to measure accuracy at both the input and output levels in a single system. The local bend network is a system in which these measurements can be made (Lewis, 1997).

\section{The local bend uses a Continuous behavioral strategy}

We have cast the results of our analysis in the context of two contrasting behavioral strategies: a Continuous strategy, in which bend direction varies continuously with touch location, and a Categorical strategy, in which one of four distinct bends is elicited, depending on which body wall quadrant the touch is given. Our data describing the input-output relationship in the local bend suggested that the Continuous strategy is most consistent with the local bend behavior, but to strengthen this argument, we performed two additional analyses. First we investigated a simple model, based on the known anatomy and physiology of the identified longitudinal motor neurons and their muscle innervation patterns. This analysis showed that the activation of the $\mathrm{D}, \mathrm{V}$, and Lat classes of motor neurons was incompatible with the Categorical strategy. However, both the D and V motor neurons (cells 3 and 4) are known to be activated substantially by a local bend stimulus (Lockery and Kristan, 1990a).

Second, we performed a series of two-stimuli experiments to test the prediction of the Categorical strategy, that the response to both stimuli would be the same as that produced by either one of the stimuli given individually (i.e., one response would win). The experiment showed that the responses to two stimuli was better described by an average of the responses to the individual stimuli. This result is consistent with the Continuous strategy. Taken together, our results suggest that the local bend behavioral output varies continuously with touch location.

\section{Population coding for Continuous and Categorical behaviors}

Several systems can be related directly to the discussion of Continuous and Categorical behavioral strategies. The turtle scratch

$\leftarrow$

gray arrow). C, Comparison of the location of the predicted and actual peak responses to two stimuli for both behavioral strategies. The prediction for the Continuous strategy ( filled circles) is given by the vector sum of the responses to $S 1$ and $S 2$, and the prediction for the Categorical strategy (unfilled squares) is the average response to $\mathrm{S} 1$ alone (note that this response does not always correspond to the ideal of $-135^{\circ}$ ). The predictions in each case are plotted against the measured mean response (six animals, three to six trials each). A point falling on the identity line corresponds to a perfect prediction. 
reflex (Stein, 1989) also can be considered Categorical, in that one of three distinct forms of the behavior is elicited, depending on the stimulus location (touch to the body surface). This is not strictly true, because there are transition regions where stimuli can evoke blends of the different behavioral forms (Mortin et al., 1985). Directed saccadic eye movements in primates clearly are governed by a Continuous strategy (Lee et al., 1988). The strategy used to produce wind-evoked escape responses in crickets and cockroaches (Miller et al., 1991; Tauber and Camhi, 1995) is more difficult to determine. Many studies of the behavior have been aimed at the problem of right-left discrimination, a categorical task (Levi and Camhi, 1996). Tauber and Camhi (1995) characterized the behavioral responses to a broader range of wind directions, but the variability in the response makes its difficult to distinguish between Categorical and Continuous strategies. At the level of the interneurons, sufficient information about wind direction is encoded to enable a Continuous strategy (Theunissen and Miller, 1991).

In each of these systems, interneurons involved in the behavior are broadly tuned to the stimulus input (Miller et al., 1991; Berkowitz and Stein, 1994), suggesting that the stimulus is represented as a population code. Microstimulation and inactivation of neurons in the superior colliculus produce effects on saccadic eye movements that provide direct evidence for population coding in this system (Lee et al., 1988). Similarly, in the local bend network, the mechanosensory neurons are broadly tuned to touch location (Nicholls and Baylor, 1968; Lewis, 1997). Electrophysiological studies also have revealed broad tuning and distributed synaptic organization of the local bend interneurons (Lockery and Kristan, 1990b). This suggests that the local bend network also uses population coding. It is an open question, in all of these systems, how the population-coded sensory stimulus is translated into the appropriate motor command.

\section{Choosing between behaviors}

The choice of behavioral form in Categorical behaviors implies that at some level in the sensorimotor pathway population-coded information must be channeled into appropriate categories. This process could involve the nervous system, where some competitive mechanism (e.g., mutual inhibition) between the subnetworks underlying each behavior was present. Alternatively, this process could be attributable to biomechanical constraints (see Levi and Camhi, 1996). A simple example of behavioral choice by non-neural mechanisms is suggested in Figure $9 B$ of the present study. If a system were constructed as in this example, the motor neurons could be broadly tuned to a stimulus, but a categorical response would be produced because of the pattern in which these motor neurons innervate the muscle.

A standard approach in the study of behavioral choice is to deliver a stimulus that alone elicits one specific behavior, along with a conflicting stimulus that alone produces another behavior. The response to such two-stimuli protocols depends on the underlying organization of the behaviors in question. In some cases, one or the other behavior will dominate (Shaw and Kristan, 1997), corresponding to a pure Categorical strategy. In other cases, a dominant behavior is not always evident. For example, in the turtle scratch reflex, the two-stimuli protocol occasionally produced blends of two behavioral forms (Stein et al., 1986); the most common response in these experiments, however, was one or the other behavior. This suggests a modular organization in the networks underlying scratching, with each module acting independently but not always in a mutually exclusive manner. The results we present here for the two-stimulus protocol eliminate the possibility that the local bend is a pure categorical behavior. However, we cannot discount a model consisting of four independent modules that can act together to form blends. Indeed, for the local bend, such a modular organization could form a cartesian representation of motor output and thus could be a simple way to produce a continuously varying response.

In conclusion, the local bend network solves two interesting problems from the perspective of neural computation. First, the mechanosensory neurons form an accurate representation of touch location. Second, the subsequent neuronal levels compute the transformations that result in behavioral output that varies continuously with touch location. These processes apparently involve neuronal population coding; however, the detailed cellular and network mechanisms are not known. This study provides a necessary context for further work aimed at elucidating these mechanisms. The relative simplicity of the local bend behavior and its underlying neuronal networks, as well as the experimental accessibility of the system, provides a unique opportunity to investigate sensorimotor transformations in a detailed and quantitative manner.

\section{REFERENCES}

Batschelet E (1981) Circular statistics in biology. New York: Academic. Berkowitz A, Stein PS (1994) Activity of descending propriospinal axons in the turtle hindlimb enlargement during two forms of fictive scratching: broad tuning to regions of the body surface. J Neurosci 14:5089-5104.

Chatterjee S, Price B (1991) Regression analysis by example. New York: Wiley.

Churchland PS, Sejnowski TJ (1992) The computational brain. Cambridge, MA: MIT.

Gu XN (1991) Effect of conduction block at axon bifurcations on synaptic transmission to different postsynaptic neurones in the leech. J Physiol (Lond) 441:755-778.

Katz PS (1996) Neurons, networks, and motor behavior. Neuron $16: 245-253$.

Knudsen EI, Blasdel GG, Konishi M (1979) Sound localization by the barn owl (Tyto alba) measured with the search coil technique. J Comp Physiol [A] 133:1-11.

Knudsen EI, du Lac S, Esterly SD (1987) Computational maps in the brain. Annu Rev Neurosci 10:41-65.

Krasne FB, Wine JJ (1984) The production of crayfish tailflip escape responses. In: Neural mechanisms of startle behavior (Eaton RC, ed). pp 179-212. New York: Plenum.

Kristan Jr WB (1982) Sensory and motor neurones responsible for the local bending response in leeches. J Exp Biol 96:161-180.

Kristan Jr WB, Stent GS, Ort CA (1974) Neuronal control of swimming in the medicinal leech. I. Dynamics of the swimming rhythm. J Comp Physiol [A] 94:97-119.

Kristan Jr WB, Lockery SR, Lewis JE (1995) Using reflexive behaviors of the medicinal leech to study information processing. J Neurobiol 27:380-389.

Lee C, Rohrer WH, Sparks DL (1988) Population coding of saccadic eye movements by neurons in the superior colliculus. Nature 332:357-360.

Levi R, Camhi JM (1996) Producing directed behaviour: muscle activity patterns of the cockroach escape response. J Exp Biol 199:563-568.

Levin S, Pearsall G, Ruderman RJ (1978) Von Frey's method of measuring pressure sensibility in the hand: an engineering analysis of the Weinstein-Semmes pressure aesthesiometer. J Hand Surg (Am) 3:211-216.

Lewis JE (1997) From touch localization to directed behavior: neural computation in the leech. PhD thesis, University of California, San Diego.

Lewis JE, Kristan Jr WB (1996) Somatosensory information processing in the leech local bend network: stimulus encoding and behavior. Soc Neurosci Abstr 22:1082.

Lockery SR, Kristan Jr WB (1990a) Distributed processing of sensory information in the leech. I. Input-output relations of the local bending reflex. J Neurosci 10:1811-1815. 
Lockery SR, Kristan Jr WB (1990b) Distributed processing of sensory information in the leech. II. Identification of interneurons contributing to the local bending reflex. J Neurosci 10:1816-1829.

Lockery SR, Kristan Jr WB (1991) Two forms of sensitization of the local bending reflex of the medicinal leech. J Comp Physiol [A] 168:165-177.

Mason A, Kristan Jr WB (1982) Neuronal excitation, inhibition and modulation of leech longitudinal muscle. J Comp Physiol [A] 146:527-536.

Miller JP, Jacobs GA, Theunissen FE (1991) Representation of sensory information in the cricket cercal sensory system. I. Response properties of the primary interneurons. J Neurophysiol 66:1680-1689.

Mortin LI, Keifer J, Stein PSG (1985) Three forms of the scratch reflex in the spinal turtle: movement analysis. J Neurophysiol 53:1501-1516.

Muller KJ, Nicholls JG, Stent GS (1981) Neurobiology of the leech. Cold Spring Harbor, NY: Cold Spring Harbor Laboratory.

Nicholls JG, Baylor DA (1968) Specific modalities and receptive fields of sensory neurons in CNS of the leech. J Neurophysiol 31:740-756.

Salinas E, Abbott LF (1995) Transfer of coded information from sensory to motor networks. J Neurosci 15:6461-6474.

Shaw BK, Kristan Jr WB (1997) The neuronal basis of the behavioral choice between swimming and shortening in the leech: control is not selectively exercised at higher circuit levels. J Neurosci 17:786-795.

Stein PS (1989) Spinal cord circuits for motor pattern selection in the turtle. Ann NY Acad Sci 563:1-10.
Stein PS, Camp AW, Robertson GA, Mortin LI (1986) Blends of rostral and caudal scratch reflex motor patterns elicited by simultaneous stimulation of two sites in the spinal turtle. J Neurosci 6:2259-2266.

Stuart AE (1970) Physiological and morphological properties of motoneurones in the central nervous system of the leech. J Physiol (Lond) 209:627-646.

Tauber E, Camhi JM (1995) The wind-evoked escape behavior of the cricket Gryllus bimaculatus: integration of behavioral elements. J Exp Biol 198:1895-1907.

Theunissen FE, Miller JP (1991) Representation of sensory information in the cricket cercal sensory system. II. Information theoretic calculation of system accuracy and optimal tuning-curve widths of four primary interneurons. J Neurophysiol 66:1690-1703.

Wilson RJA, Skierczynski BA, Blackwood S, Skalak R, Kristan Jr WB (1996) Mapping motor neurone activity to overt behaviour in the leech: internal pressures produced during locomotion. J Exp Biol 199:1415-1428

Yau KW (1976) Physiological properties and receptive fields of mechanosensory neurones in the head ganglion of the leech: comparison with homologous cells in segmental ganglia. J Physiol (Lond) 263:489-512.

Yuille AL, Grzywacz NM (1989) A winner-take-all mechanism based on presynaptic inhibition feedback. Neural Comput 1:334-347. 